\section{Editorial}

B ajo este título recogen los arquitectos Fuensanta Nieto y años en una reciente monografía. Entre estos dos conceptos, complementarios antes que opuestos, discurre la entrevista
que publicamos en las primeras páginas de esta revista, y que construye precisamente la pregunta que formulan en la Una selección de las mejores aportaciones será realizada por los revisores de la revista y presentada en un acto público que
recorrerá en directo todos los contenidos de las distintas secciones de la revista y que se celebrará entrada la primavera de

Tradición y originalidad es la versión próxima, pero sin duda no idéntica, de estos dos polos entre los que se mueve según
Kenneth Frampton la joven arquitectura española de princip--
ios de este siglo. Conceptos retomados en este texto inédito ios de este siglo. Conceptos retomados en este texto inéd
transcrito por Palimpsesto e introducido por Jesús Donaire, comisario junto a Jesús Aparicio de la exposición "Young
Architecture of Spain" que cierra este año una itinerancia por 18 ciudades de todo el mundo.

Como una de las más recientes figuras de esta generación de arquitectos Olga Felip, que junto a Josep Camps forma estudio profesional, nos desentraña en su texto-reseña de
la conferencia impartida en la Cátedra Blanca de la ETSAB los mecanismos empleados en su proceso de proyecto. La distintas ideas, como Godard en el film "Vivre sa vie".

Este procedimiento, próximo al montaje en seco, conduce la al origen de las cosas, Cristina Pardal, con la introducción de Ignacio Paricio, realiza una necesaria reubicación en la arquitectura contemporánea de los conceptos de fachada
ventilada, drenada y revestida. La confusión en el empleo de estos términos conduce a praxis equivocadas e innecesarias.

La reflexión sobre las envolventes, de nuevo entre lo adquirido y lo propositivo, encuentra en el corazón de la revista uno
de sus temas centrales, la ventana. La cuidadosa selección por parte de Carlos Ferrater de seis ventanas de otros tantos maestros de la historia de la arquitectura, además de reivindi-
car su pertinencia frente a lo ligero, ilustra cómo es posible el progreso desde la historia. Seis regalos que aguardan com-
pletarse con las colecciones particulares de cada lector.

El matiz, la variación sobre un mismo tema, conduce nuestra narración a la aportación de Álvaro Clúa titulada "La arquitectu-
ra del swing" y surgida de la convocatoria realizada por Alvaro
Siza en el número 9. Pequeñas variaciones de la partitura permiten adaptaciones, integraciones, matices, precisamente "Bono ocurre en el proyecto de Siza en Berlin conocido como dad urbana en el proyecto de Mario Ridolfi y Wolfgang Frank proyecto, fruto de la reconstrucción tras la guerra, nos remite a neorrealismo italiano y al interés que este tipo de posicionami-
entos suscita en la actualidad. Ferran Grau en su tesis doctoral y actualiza el concepto de Realismo Crítico en la era de la simultaneidad. Lo hace a través de una serie de proyectos, definitiva el verdadero lenguaje del arquitecto.

Lenguaje que el arquitecto y poeta Joan Margarit, en la conversación que mantuvimos con él, declina en palabras precisas, siempre que, en su mano, como los trazos de sus estructuras y de sus poemas, ex
condición humana.

PALIMPSESTO

\title{
Fuensanta Nieto y Enrique Sobejano
}

Entrevista realizada en el despacho de los arquitectos en Madrid el 8 de octubre de 2013. Alberto Peñín

La conversación se inicia con Enrique Sobejano, a la que se incorpora Fuensanta Nieto en un trajín de proyectos, maquetas y sus desplazamientos a Berlín. Acaban de recibir su monodel Call for papers propuesto para próximo número de la revista.

\section{Formación}

¿Por qué decidisteis dedicaros a la arquitectura?

En mi caso la motivación no fue familiar, puesto que soy el primero de toda la rama, ni por relaciones cercanas. Tampoco fue algo muy temprano, que tuviera claro desde la infancia. Lo mío fue más tardío y no muy consciente, al comienzo, de lo que suponía esa decisión.

¿Cómo fue vuestra formación?

De igual modo que hay arquitectos que se han sentido muy cercanos a algún maestro que les ha abierto el camino, en nuestro caso no fue así. La Escuela de Madrid supuso una buena formación, cosa que ahora puedo distinto a desde un punto de vista distinto al estar dando clases en una directamente guiados por ningún arquirectamente guiados por ningun arpor lo que indirectamente aprendimos y conocimos, es decir por aquellos que podríamos considerar padres de que podriamos considerar padres de sido alumno de ellos, Saénz de Oiza y De la Sota fueron arquitectos que me influyeron claramente. Realmente son, junto con Coderch, los tres arquitectos que muchos de nosotros tuvimos tos que muchos de nosotros tuvimos como referencia, aunque no fuera di recta.

En cambio, al acabar la carrera, al ir a los Estados Unidos, -donde Fuensanta y yo nos "reconocimos"- se abrieron muchos caminos para nosotros, iguras que te mencionaba.

Recuerdo que precisamente Kenneth Frampton lo primero que nos preguntó al ver que éramos españoles fue acerca de toda esta arquitectura de los años 60. Nos hablaba de los poblados dirigidos, de Caño Roto de Vázquez de Castro, por ejemplo. Sin duda nos influyeron mucho aquellos dos años en Estados Unidos.

¿Y qué es lo que crees que pudo aportaros aquella estancia?

Nos aportó una visión exterior de la disciplina. Algo que nos hizo comdisciplina. Algo que nos hizo comque habíamos aprendido en la escuela en Madrid. La universidad americana es más dada al debate teórico y quizá la española estaba más atenta a lo proyectual y constructivo. También descubrimos cuestiones muy útiles tales como aprender a hablar en público, o a comprender que la arquitectura abarca campos muy amplios, más allá de la simple elaboración de unas plantas, alzados o secciones. La arquitectura es compleja y es esencial saber transmitirla, trans

Volviendo a la influencia de los maestros, Ilama la atención el acento puesto sobre Utzon y en particular sobre su proyecto para el museo de Silkeborg del que editaste un texto, que es tal vez uno de los más prto, que es talvez uno de los más proposino , inclus enterrado en aquellas geometrías...

Utzon es uno de los arquitectos de Utzon es uno de los arquitectos de
los que hemos aprendido constantemente, a través de trabajos, publicamente, a través de trabajos, publicacasi todas sus obras. Es un arquitecto que abre muchísimos caminos aunque en muchos casos no llegaran al final. El museo de Silkeborg, por ejemplo, pero también muchos otros, incluso sus esbozos, sus ideas.

engo que decir que el primer trabajo que nos pidió Frampton en la Universidad de Columbia fue sobre Utzon. Hay que pensar que en la Escuela de Madrid estaba casi olvidado cuando estudiábamos, excepto para Oíza o Fullaondo. Cuando yo acabé en 1981 no formaba parte del discurso arquitectónico del momento, más atento a los inicios del postmodernismo y la discusión sobre la historia. Utzon era discusión sobre la historia. Utzon era un arquitecto que no entraba en la enseñanza de aquellos años. Por ejemplo, en la Historia de la Arquitectura a lo sumo, aparecía como una nota a a lo sumo, apare

pie de página. Como decía, fue Frampton quién nos hizo fijarnos en su obra. Más tarde, cuando comenzamos a dirigir la revista "Arquitectura" y, tras un primer número centrado en Le Corbusier, dedicamos el segundo a Utzon con

P Proceso creativo

Cómo es vuestro proceso creativo? ¿Abordáis conjuntamente quella hoja en blanco a la que aludía Oíza?

No creemos que haya una hoja en blanco. Siempre existe algo previo, datos, la memoria de un lugar y nuestra propia experiencia. No solo me refiero a las vinculaciones con el lugar, si no a la relación que los proyectos y obras establecen entre sí. De hecho, nuestro libro Memory and Invention, que hoy mismo hemos recibido, se nicia con tres citas, una de la cuales, de Georges Perec, dice: "Toda obra fica al Atlas Mnemosyne de cita gráburg d nas Marentre diferentes obras. Los proyectos, entre diferentes obras. Los proyectos, res viajes, nuestros recuerdos, interfiede que la hoja en blanco no exista, porque de hecho no existe. La mente no está nunca en blanco.

Cuál es el momento de vuestro proceso de mayor tensión creativa? Q Qué papel juegan las maquetas en la elaboración de las propuestas?

No existe un momento determinado, xisten múltiples. A veces son cuestiones secundarias las que ponen en marcha el proyecto.

hasta que no construimos una maqueta, aunque sea rápida y de cartón o acabamos de ver lo que estamos haciendo.

La idea arquitectónica ha de ser reconocible y transmisible? ¿Los mecanismos de proyecto deben hacerse evidentes?

Cuando existe una idea, que es siempre más universal, se transmite fácilmente. Pero cuando hablamos de mecanismos o procesos, éstos son més personales. Se ha extendido en os últimos años una cultura arquiectónica que ha puesto en valor el proceso por encima del resultado. Continuamente aparecen análisis, críticas, conferencias o debates que hablan de los procesos de proyecto. A mí me interesa entender el proceso con una perspectiva temporal, es ecir como un conjunto de resultados parciales.

\section{Presencia}

En una ocasión hablabais de la depuración de lo innecesario hasta alcanzar la raíz mínima de la arquitectura, recurriendo a la poesía visual. ¿Es tal vez ese trabajo de síntesis arquitectónica el que logra or otra vía una cierta desaparición del arquitecto?

En nuestro libro citamos una poesía visual en la que se suprimen sucesivaal límite en el que el texto deja de ser comprensible. Esta metáfora representa una aspiración común a la mayor parte aspiración común a la mayor partes trabajo, es decir pero sin llegar a perder el significado del proyecto.

Nos interesan las metáforas entendidas como "leit motiv" que guían todo el proceso. Un ejemplo seria el proyecto, para nosotros quizá esencial, de Medina Azahara. Allí la idea o la metáfora no consiste en una imagen, sino más bien en una determinación "trabajaremos como arqueólogos". A partir de esa premisa, todo lo que viene después, desde el proyecto hasta la obra, queda incorporado a aquella intención. En otras palabras, e edificio aparece bajo tierra, el materia es hormigón puesto que una vez desencofrado ya está acabado, el color es blanco como el de la antigua ciudad árabe, etc.

No creemos que haya una boja en blanco. Siempre existe algo previo, datos, ta memoria de un lugar y nuestra propia experiencia. No solo me refiero a las vinculaciones con el lugar, s no a la relación que los proyec tos y obras establecen entre sí.

Cuando os enfrentáis a una realidad construida ¿facilita una suerte de contextualización del proyecto? ¿Sería lógico que la mano del arquitecto tendiera a diluirse?

En nuestra opinión, hay proyectos en los que la mano del arquitecto debe desaparecer casi del todo. El Museo Nacional de Escultura que rehabilitamos en Valladolid supuso un esfuerzo enorme para que la mayor parte de las operaciones en un edificio complejo y de gran tamaño no fueran visibles. Tenía sentido que fuera así, porque e edificio histórico era de gran valor, y habia conservado en gran medida sus elementos arquitectónicos.

En otros casos, como el museo de Moritzburg, donde quedaba solo la ción de sus muros, nuestra intervención no solo es visible sino que se convierte en protagonista. Establecemos un diálogo entre la intervención No nos interesa oposición original. No nos interesa la oposición canónica de lo nuevo y lo antiguo que el mo- 


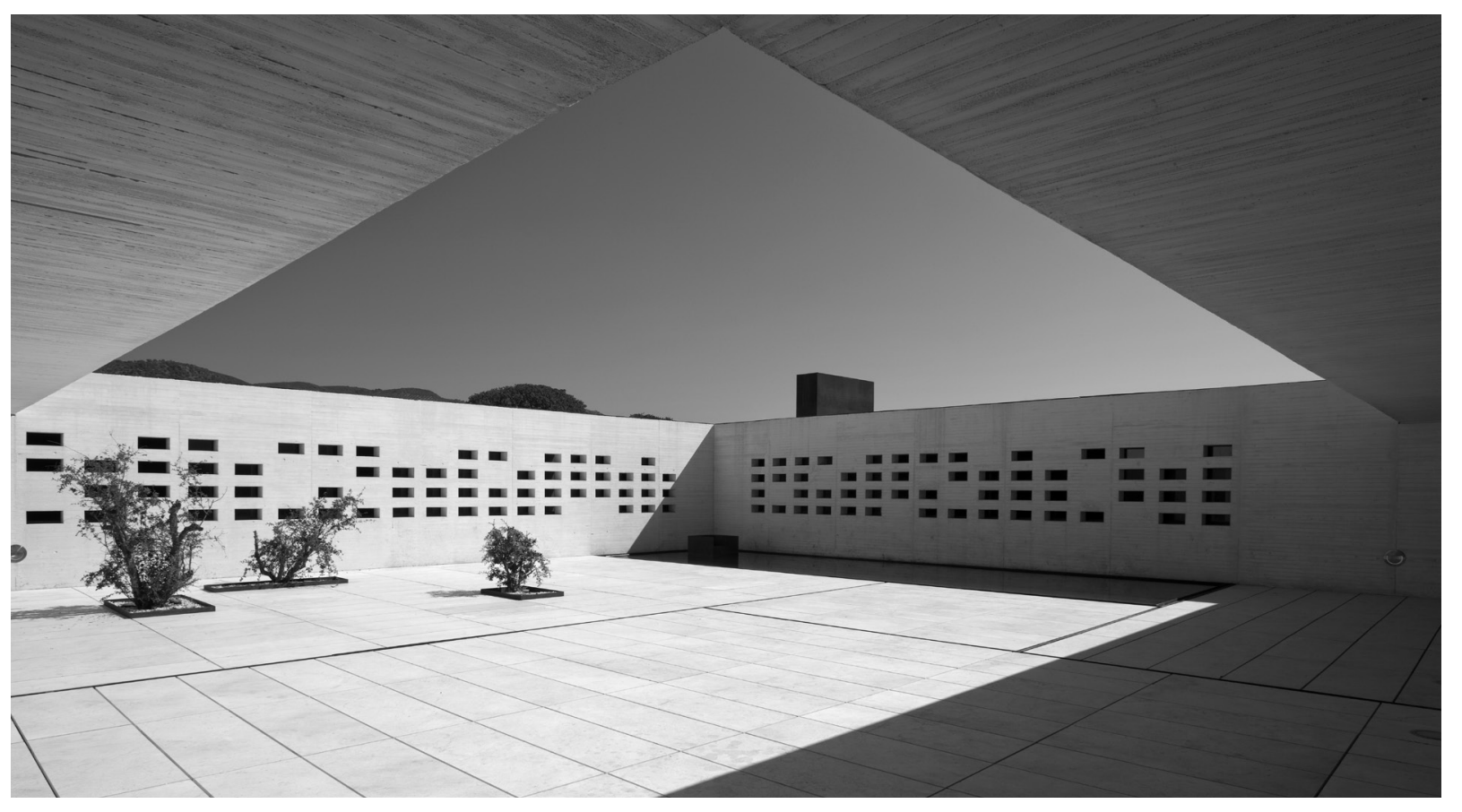

Museo Madinat Al Zahra (Fotografía Fernando Alda)

\section{Combinatoria}

En alguna ocasión mencionabais el origen de vuestra arquitectura en el cruce de las preexistencias con ciertas invariantes como la luz, o la geometría. En vuestro caso parece que se recurra a una geometría sencilla que, además de facilitaros el control del proyecto, os permite abordar la complejidad, y no hablo de una geometría parametrizada y no aprehensible.

Si observamos lo que nos rodea, todo puede entenderse como un sistema combinatorio que finalmente se limita a las variaciones de un número muy reducido de elementos. Así sucede en todos los ámbitos, en un partitura musical con la combinación de 12 tonos, en un texto escrito, con 27 letras, y así sucesivamente: tres colores primarios en la pintura, cuatro bases genéticas en el ADN de los seres vivos, etc. Todo es pues combinatoria. En nuestros proyectos tratamos de conducir nuestro esfuerzo hacila la combinación de los minmos elementos posibles, en las múliples varaciones aue evitan la simple repetición. Los programas informálicos ayudan, sin duda, pero para nosotros siempre entran en el proceso más tarde, una vez hemos tratado de comprender los elementos con los que trabajar.

Cuando trasladáis la repetición y la variedad a lo constructivo aparecen paneles de fachada, variedades en texturas; Mérida, San Telmo, Córdoba... Parece como si la prefabricación fuera la traducción a lo constructivo de la repetición y la "customización" de la variedad.

Esta es otra cuestión importante, es decir, el valor expresivo del material y la manera en la que éste se transforma en un sistema industrializado, con muchas posibles variaciones. Nos interesa la relación entre el objeto que surge de una intuición, a veces de una obra única y personal, con el proceso que conduce a su prefabricación.

Esta secuencia la hemos desarrollado de distintas modos en diferentes proyectos, a menudo en colaboración con artistas. En el auditorio de Mérida trabajamos con la escultora Esther Pizarro, que realizó un relieve original que no medía más que 45 por $15 \mathrm{~cm}$. A partir de ahí sus combinaciones construyen fachad a mayor escala en paneles prefabricados construyen la También operamos de similar modo en el palacio de hormigon. Ta recientemente en la fachada del Centro de Arte de Córdoba.

En nuestros proyectos tratamos de conducir nuestro esfuerzo hacia la combinación de los mínimos elementos posibles, en las múltiples variaciones que evitan la simple repetición.

\section{P Arte y programa}

Volviendo a vuestra experiencia americana y en relación a otras disciplinas vinculadas a la arquitectura, ¿cuáles creéis que tienen cabida en la génesis del proceso de proyecto? ¿Las artes visuales en particular?

Yo diría que así ha sucedido en numerosas ocasiones. En mi caso estoy dando clases en Berlín en la Universidad de las Artes (UdK) lo cual me resulta especialmente interesante por las interferencias que se dan, a veces inconscientemente, entre las distintas artes que allí confluyen. Los estudiantes de arquitectura tienen la posibilidad de un diálogo constante con los artistas. Esa fue también nuestra actitud al decidir colaborar en el Palacio de Congresos de Mérida con Esther Pizarro, en el museo de San
Nos interesa la relación entre el objeto que surge de una intuición, a veces de una obra única y personal, con el proceso que conduce a su prefabricación.

FN. En este momento estamos trabajando en Múnich, donde tro cliente finalmente es una empresa privada. El proyecto sustituye edificios de los años 60 por una nueva ordenación para oficinas y hotel. Es decir, trabajamos para un cliente privado al que hemos llegado a través de un concurso público, lo cual otorga al arquitecto mayor fuerza en el diálogo.

Es un proyecto muy complejo por estar situado en una de las entradas a la ciudad, cerca del centro. Lo interpretamos como un puerta de cceso a Múnich. El concurso implicó un diálogo desde el principio, con un jurado muy numeroso en un dialogo ban representados la ciudad, los clientes, arquitectos que estatigio, los partidos políticos en el poder y en el oposición, urbanistas y técnicos.

ES. Se trata de un caso extremo. Mucha edificabilidad, distintos clientes, condiciones de todo tipo, varias fases... Y ante esta situación, propusimos actuar de nuevo con un sistema combinatorio. Proyectamos una única planta, rotada en distintos ángulos, con diferentes alturas, y techos inclinados en varias direcciones. Es un esfuerzo de economía conceptual que se traslada a la economía real, en los detalles, sistemas constructivos,
estructurales, etc. Estamos en plena definición del proyecto en un proceso muy distinto al de aquí en España.

\section{Definición material}

Telmo de San Sebastián, con Leopoldo Ferrán y Agustina Otero, o en Cordoba con "Realities United".

En los tres casos estoy convencido de que la colaboración ha ido en favor de un proyecto de mucho mayor interés. Tanto desde el punto de vista de los artistas, que se encuentran en muchas ocasiones sin la posibilidad de intervenir en las escalas de la arquitectura, como en el de los arquitectos, que a veces podemos estar demasiado influidos por las limitaciones económicas, técnicas y normativas, que nuestro trabajo lleva implícito.

Trabajar en ese límite, en esa franja donde arte y arquitectura coinciden, nos interesa mucho.

Otra cuestión importante es el valor expresivo del material y la manera en la que éste se transforma en un sistema industrializado, con muchas posibles variaciones. Nos interesa la relación entre el objeto que surge de una intuición, a veces de una obra única y personal, con el proceso que conduce a su prefabricación.

El tipo de programas, ¿no facilita una aproximación al proyecto desde la autonomía de la arquitectura? Después de Europan, ¿tenéis algún proyecto de vivienda en el tablero?

Reconozco que no, si bien sí hemos tenido la oportunidad de construir algunas recientemente, por ejemplo el lugar donde estamos, nuestro estudio, forma parte de una rehabilitación en la que se ha transformado una nave en viviendas, y ahora mismo estamos terminando otro pequeño proyecto en la ciudad de Madrid. En cuanto a la vivienda social, tuvimos la oportunidad de trabajar hace muchos años, en una escala importante como fue el proyecto de Sevilla para Europan. Pero, ¿hasta qué punto uno elige sus programas? Nunca totalmente, aunque de alguna manera los buscamos, puesto que nuestra obra se desarrolla casi toda a través des, puesto que nuestra obra se desarrolla sentarse o no.

Se incorpora Fuensanta Nieto a la conversación.
¿En qué momento de ese proceso interviene la construcción, la definición material?

ES. En el caso de nuestros proyectos en Alemania, la construcción y la definición material entra desde el comienzo. Está interde modo que cada decisión está siendo valorada económica y técnicamente. Este procedimiento llega a un extremo muy diferente al que estamos acostumbrados en España, en donde habitualmente el arquitecto toma muchas decisiones por su cuenta sin necesidad de consensuarlas al principio. Las condiciones técnicas y normativas inciden en todo momento del proceso.

Precisamente en relación al carácter colectivo de nuestro trabajo y la especialización, ¿cómo trabajáis con los ingenieros? ¿Intervienen en las fases iníciales del proyecto?

ES. Con los ingenieros de estructuras en ocasiones sí, pero hemos de reconocer que en otras especialidades suelen entrar después. De alguna manera la forma, la estructura y la construcción forman parte de la concepción espacial. Cada vez más a menudo, las cuestiones ligadas a la sostenibilidad y al medio ambiente forman parte también de la fase de concepción.

Javier Manterola nos decía "hace unos números" que el arquitecto se ocupaba del mundo de la compresión, de lo grávido, mientras que el ingeniero tenía menos prejuicios al respecto y atendería por igual a las cuestiones de la tracción. ¿Os reconoceríais en esta lectura?

Esa frase resume la propia esencia de la arquitectura entendida como la oposición entre el deseo de anclarse a un lugar, a un terreno (en el origen, una cueva), frente a la voluntad de superar festado con mayor claridad. La arquitectura gótica, por ejemplo, trata de "vencer" la gravedad, o la arquitectura japonesa busca la ligereza. La arquitectura romana, por el contrario, podríamos oposición existe siempre, varía el grado en el que una posición porque pensamos que cada proyecto requiere una interpretación particular. Algunos de nuestros proyectos buscan la ligereza, mientras que otros requieren peso. viniendo en todo. Aún estamos en la fase de concepto y ya estamos colaborando con un equipo muy grande de consultores, la gravedad. En ciertos momentos en la historia esto se ha manidecir que es masiva, apegada al terreno, a la gravedad. Esa domina sobre la otra. Nosotros no nos adscribimos a ninguna

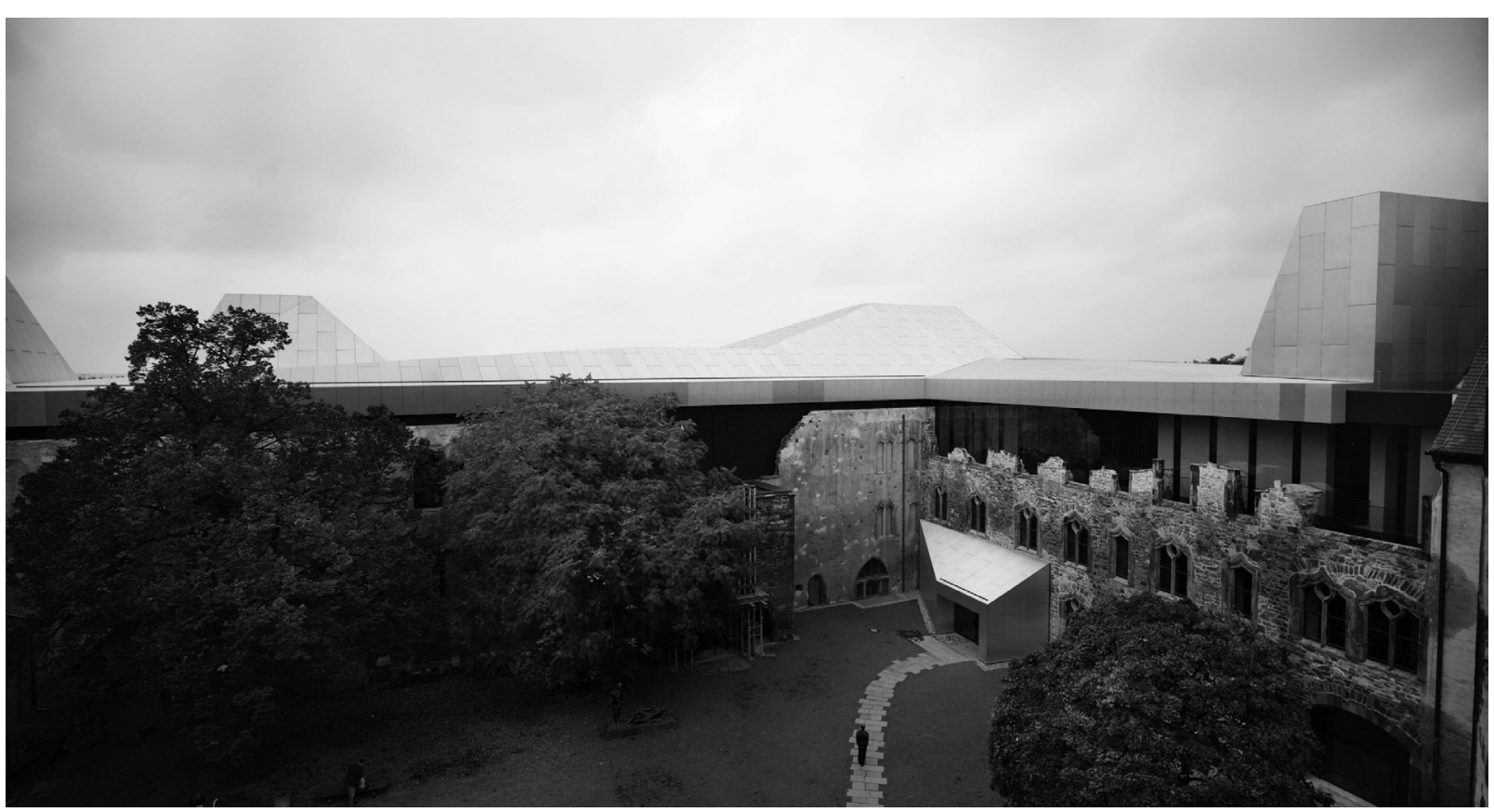




\section{Arquitectura y sociedad}

El reconocimiento internacional de nuestra arquitectura coincide con dudas sobre nuestra valoración en casa, social y no digamos política. Más allá de Esperanza Aguirre y de la LSP, ¿qué hemos hecho mal?

FN. Quizá hemos transmitido mal la imagen de nuestro trabajo. Probablemente se nos identifica a todos con algunos arquitectos que no han tenido precisamente una actuación responsable en cuanto al paisaje, la escala urbana, o la calidad espacial. También sucede que en España mucha gente no distingue claramente entre la labor del arquitecto y la de otros agentes de la construcción, es decir, promotores, constructores, ingenieros, etc. Cuando algo sale mal -y en nuestro país se han hecho, junto a obras de gran calidad, muchas otras fallidas- se tiende a personalizar en el arquitecto todos los errores que se producen en una obra. Es algo que seguramente nosotros no hemos sabido explicar bien.

ES. Desde luego en España la imagen del arquitecto ahora es peor que hace unos años. La paradoja es que internacionalmente está muy valorada. La arquitectura hecha con intención, dedicación y calidad no deja de ser un porcentaje pequeño, en España y en cualquier otro país. Por el contrario, hay que ser consciente de que una gran parte de las obras construidas en os últimos años ha dado lugar a la destrucción paisajística de muchas costas, a promociones abandonadas, etc.

FN. Como decía, no se diferencia muy bien en la prensa, en la a opinión de la gente, al arquitecto del resto de los agentes de de todo, cuando en muchas ocasiones no es asi. Por ejemplo uno de los problemas de los edificios es la falta de mantenimiento. Un edficio hay que mantenerlo; por muy bien que se construya, por buenos materiales que se utilicen, si no tiene manten atribuyen a los arquitectos, cuando corresponden a la falta de calidad de la ejecución, o a la carencia de mantenimiento por parte del usuario.

ES. Dado que ahora estamos entrando en cuestiones más proesionales, en nuestra opinión no entendemos esta obsesión de os colegios y consejos profesionales por que el arquitecto sea responsable de todo, de todas las ingenierías, con el argumento por el cual si perdemos esa responsabilidad, se pierde también el control de la obra. Nuestra experiencia fuera de España, por ejemplo en Alemania, Austria, Holanda, no es así. Allí no asumimos la responsabilidad de la estructura, las instalaciones, etc. aunque sí la coordinación. Con lo cual los ingenieros están contratados directamente por el cliente, con mejores honorarios, pero también con una responsabilidad muy clara. Este es otro de los problemas de nuestra profesión en España.

\section{P De lo privado}

¿Tratáis de trazar una línea entre lo privado y lo profesional, o la arquitectura es vuestra manera de vivir?

ES. Es inevitable pensar que en nuestro caso es una manera de vivir. Cuando viajas, estás viendo arquitectura, cuando lees, o ves una película, no puedes evitar establecer relaciones con la arquitectura. Tratamos, no obstante, de que no se convierta en obsesiva y llevarlo de una forma natura.

FN. Es una forma de vida pero también se da en otras profesiones, desde en un abogado hasta en un médico...

ES. No estoy tan de acuerdo, se da sólo en aquellos a los que es gusta mucho lo que hacen se transforma en una forma de vida cuando se disfruta haciendo el trabajo.

FN. Los profesionales que viven su profesión hacen de ella una forma de vida. Creo que realmente si eres capaz de incorporar el mundo que hay alrededor de la arquitectura, no es un problema.

No entendemos esta obsesión de los colegios y consejos profesionales por que el arquitecto sea responsable de todo, de todas las ingenierias, con el argumento por el cual si perdemos esa responsabilidad, se pierde también el control de la obra. Nuestra experiencia fuera de España no es asi.

\section{Teoría y práctica}

Veo que vuestro libro está estructurado no tanto por tipologías o cronologías sino en base a determinados conceptos. En vuestra opinión, ¿la teoría emana de la praxis? ¿Existe un corpus previo?

ES. Esta ha sido siempre es una cuestión de debate, la relación entre teoría y práctica. En nuestro caso, la reflexión sobre la arquitectura va paralela a la práctica como actitud contemporánea, frente a la tradición decimonónica, que establecía la prevalencia de la teoría sobre la práctica, que debía ser una consecuencia de aquélla.

Ambas están pues íntimamente relacionadas. Nuestro libro, "Memory and Invention" es una reflexión teórica, personal, acerca de conceptos arquitectónicos de carácter más general, surgidos a partir de nuestros propios proyectos.
¿Cómo ha evolucionado vuestro enfoque respecto a aquel primer libro, "Desplazamientos"?

ES. Están relacionados entre sí. "Desplazamientos" se planteaba como un puzzle de proyectos y obras, puestos en relación a nuestros viajes y experiencias, es decir a nuestros desplazamientos. Sugería ya un atlas, a la manera de Aby Warburg. A igual que en la obra del historiador alemán, siempre nos interesaron las relaciones visuales como un modo de entender la arquitectura. Este ha sido también el leitmotiv del segundo libro.

Hablando de teoría, de reflexión, y en cuanto a vuestra relación con la universidad ¿cómo se vincula vuestra práctica docente con la profesional? ¿Cómo es vuestra experiencia docente?

ES. En mi caso, como ya comenté, soy profesor desde hace seis años en Berlín. Tanto la ciudad, una de las más activas culseis años en Berin. Tanto la ciudad, una de las más activas culesta actividad docente on una experiencia diferente a la que vivi en Madrid anteriormente.

Sobre la enseñanza de proyectos, nos interesa transmitir aquello que hacemos en nuestra propia actividad como arquitectos. Veo que hacemos en nuestra propia actividad como arquitectos. Veo una diferencia entre lo que el profesor transmite y lo que proyecta o construye en la realidad. Esa es la clave a la hora de enseñar.

FN. Dar clase, como sabemos, nos fuerza a verbalizar nuestras deas, a ordenarlas, a transmitirlas. Permite fijarlas, y eso lleva a veces a replantearlas. En ese sentido, la docencia tiene también

Esta ha sido siempre es una cuestión de debate, la relación entre teoria y práctica. En nuestro caso, la reflexión sobre la arquitectura va paralela a la práctica como actitud contemporánea, frente a la tradición decimonónica, que establecía la prevalencia de la teoría sobre la práctica, que debía ser una consecuencia de aquélla.
Dar clase, como sabemos, nos fuerza a verbalizar nuestras ideas, a ordenarlas, a transmitirlas. Permite fijarlas, y eso lleva a veces a replantearlas. En ese sentido, la docencia tiene también una parte de interés personal, puesto que te obliga a esforzarte y cuestionar lo que haces.

una parte de interés personal, puesto que te obliga a esforzarte y cuestionar lo que haces.

Solemos cerrar la entrevista con la misma pregunta a todos los entrevistados: si fuerais directores de la Escuela de Barcelona, ¿qué centro ¿qué seria lo primsiro que hariais? Os recuerdo que dentro de poco hay elecciones así que os cedemos este
espacio de difusión...

ES. Creo que sería un necesario equilibrio entre optimismo y realismo que no lleve a las Escuelas a un camino como el que sin duda sufrieron lave a las Escuelas a un camino como el que sin duda

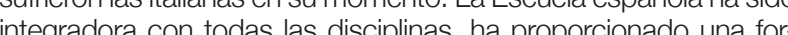
mación el proceso de proyectar ytado por una relación muy directa entre En un de proyectar y construir, entendidos como un todo. En un momento de crisis como el actual todo esto se puede deshacer. Esto sucedió en Italia y en otros países y no haría más que aumentar esta disociación entre la arquitectura y la sociedad de la que antes hablábamos.

META-ARCHITECTURE Excerpt from Memory and Invention

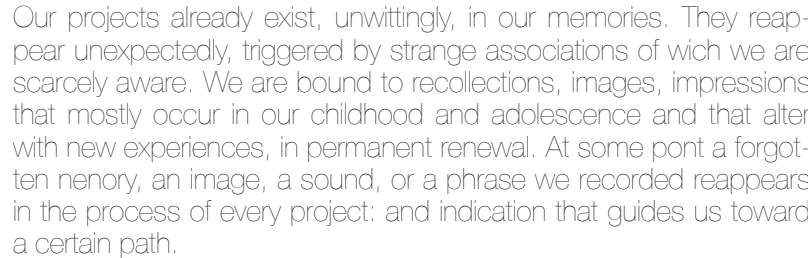

Espacio Andaluz de Creación Contemporánea (Fotografía Roland Halbe)

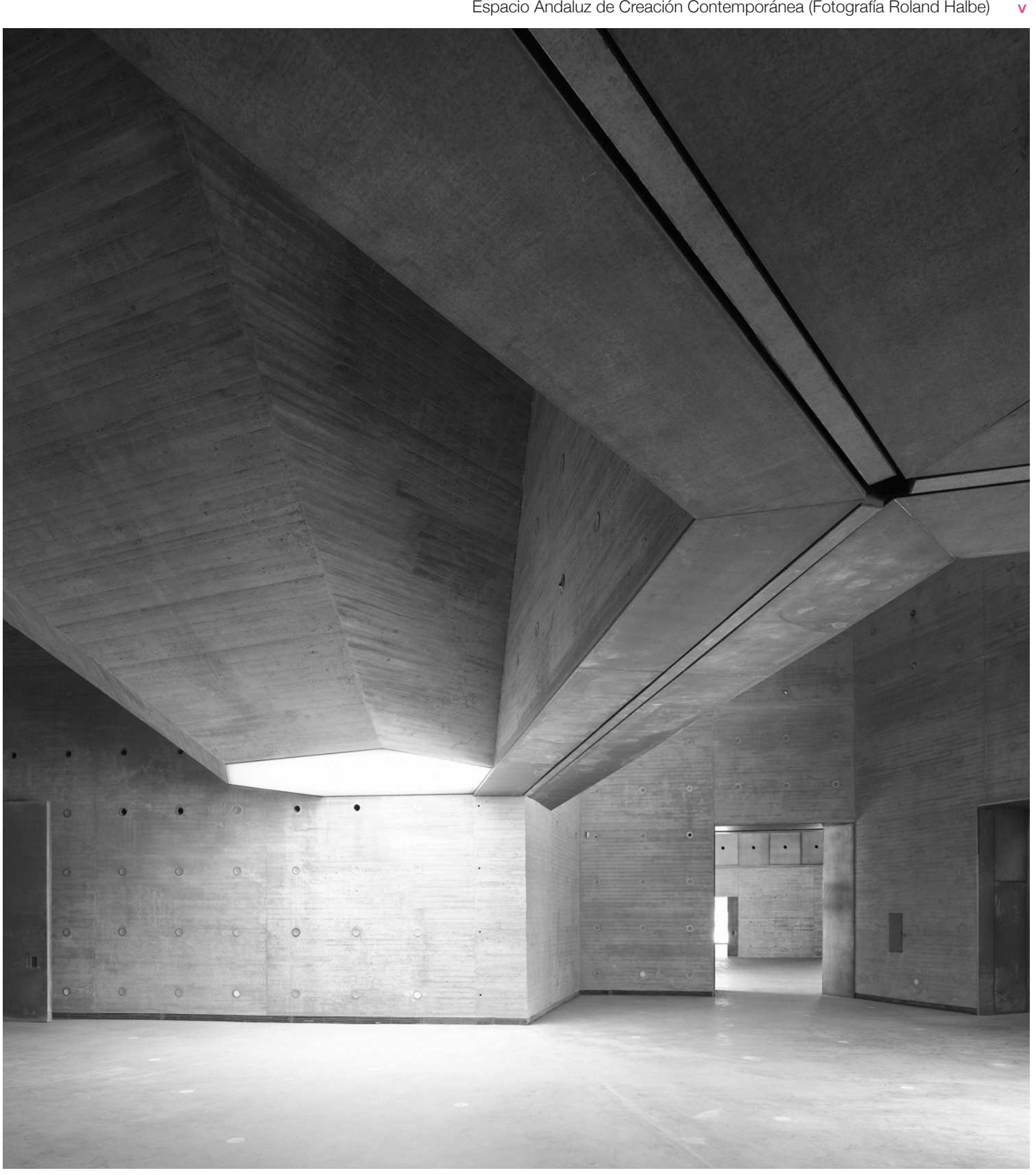

